\title{
Extraskeletal subcutaneous osteosarcoma of the upper arm: A case report
}

\author{
TOMOKI NAKAMURA ${ }^{1}$, AKIHIKO MATSUMINE $^{1}$, KEISUKE NISHIMURA $^{2}$, HIROKAZU YOKOYAMA $^{1}$, \\ TETSUYA MURATA $^{3}$, ATSUMASA UCHIDA ${ }^{1}$ and AKIHIRO SUDO ${ }^{1}$ \\ Departments of ${ }^{1}$ Orthopaedic Surgery, and ${ }^{2}$ Pathology, Mie University Postgraduate School of Medicine, Mie; \\ ${ }^{3}$ Department of Pathology and Laboratory Medicine, JA Suzuka General Hospital, Shizuoka, Japan
}

Received August 5, 2010; Accepted October 4, 2010

DOI: 10.3892/ol.2010.204

\begin{abstract}
Extraskeletal osteosarcoma (ESOS) occurs in approximately $1 \%$ of soft tissue sarcomas and $2-4 \%$ of all osteosarcomas. In particular, subcutaneous osteosarcoma is extremely rare, occurring in less than $10 \%$ of ESOS cases. This report presents a case of a subcutaneous tumor in the upper arm of a 79-year-old male. Imaging and pathological findings led to the conclusion that the soft tissue tumor should be diagnosed as subcutaneous osteosarcoma. Additionally, this case report documented the clinicopathological findings of the extraskeletal subcutaneous osteosarcoma in this case and discussed its clinical features by reviewing cases previously described in the literature.
\end{abstract}

\section{Introduction}

Extraskeletal osteosarcoma (ESOS) is a rare malignancy that accounts for approximately $1 \%$ of all soft tissue sarcomas and for 2-4\% of all osteosarcomas (1-3). ESOS usually occurs in the deep soft tissue of the extremities of adults (4). It typically arises in the deep soft tissue of the thigh. Other less frequent sites include the buttock, shoulder, trunk and retroperitoneum. Approximately $24 \%$ of cases have been associated with previous radiotherapy or trauma (5). In contrast to primary osteosarcoma of the bone, this variant typically develops after the fifth decade of life, and the prognosis is uniformly poor $(5,6)$. The present report documented the clinicopathological findings in a patient who had an ESOS arising from the subcutaneous tissue of the upper arm and reviews previous cases of subcutaneous ESOS.

Correspondence to: Dr Akihiko Matsumine, Department of Orthopaedic Surgery, Mie University Graduate School of Medicine, Edobashi 2-174, Tsu, Mie 514-8507, Japan

E-mail: matsumin@clin.medic.mie-u.ac.jp

Key words: osteosarcoma, subcutaneous tissue, extraskeletal

\section{Case report}

A 79-year-old male was referred to the Mie University Hospital due to an enlarged, slightly painful mass in the left upper arm. The patient first noted the mass 3 years prior to presentation. No history of trauma or therapy had previously occurred at this site. Moreover, the patient had experienced no recent health problems and recalled no family history of cancer. A physical examination confirmed the presence of a lobulated hard mass with a diameter of $4 \mathrm{~cm}$ at the lateral side of the left upper arm. Radiographs of the left upper arm revealed a mass with ossification which appeared to be separated from the humerus (Fig. 1). Magnetic resonance images (MRI) showed a soft tissue mass above the fascia of the triceps with a low signal intensity on T1-weighted images and a heterogeneous signal intensity on T2-weighted images (Fig. 2). Computed tomography (CT) of the chest did not demonstrate any pulmonary masses. All routine laboratory data were normal. The long clinical course from the first awareness of the tumor and the clinical findings, suggested that the tumor was benign or a malignant calcifying epithelioma with ossification. The patient was treated with wide resection and a skin graft from an inguinal lesion. Gross sectioning of the specimen showed a $4 \times 4 \times 2.5 \mathrm{~cm}$ firm and solid mass in the deep dermis and subcutaneous tissue (Fig. 3). A microscopic examination showed the presence of numerous spindle and atypical cells often exhibiting pronounced nuclear atypia or multinucleated giant cells with bone and osteoid formation. A high mitotic activity with numerous atypical mitoses was noted (Fig. 4). These findings led to the conclusion that this soft tissue tumor was a subcutaneous ESOS. The patient had no evidence of local recurrence and distant metastasis 1 year following resection.

\section{Discussion}

Since ESOS was initially described by Wilson in 1941, approximately 300 cases have been reported thus far $(5,6)$. ESOS is defined as a malignant mesenchymal neoplasm composed of cells producing osteoid, bone and/or chondroid material, with no attachment to bone or periosteum (7). It occurs most often in the deep soft tissues of the extremities of adults, at an average age of 50 years. Clinically, ESOS usually carries an extremely poor prognosis. The 5-year survival rate ranges 


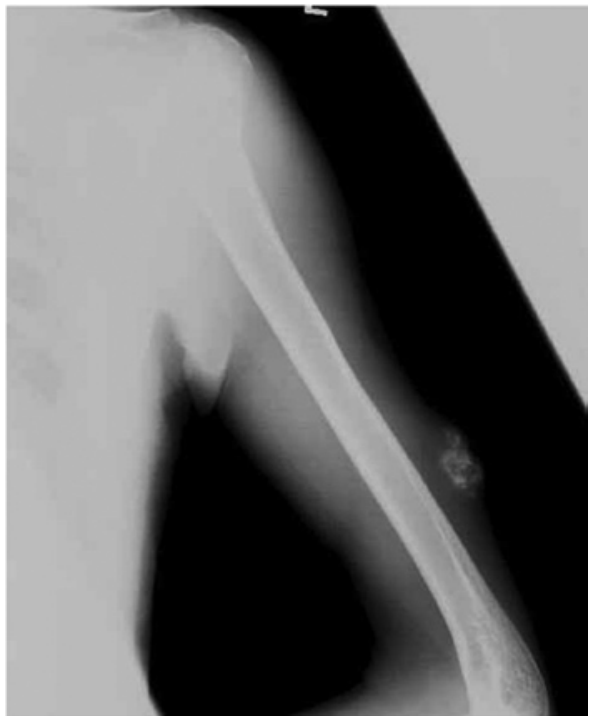

Figure 1. Radiographs revealed a subcutaneous soft tissue mass with ossification in the upper arm.

A

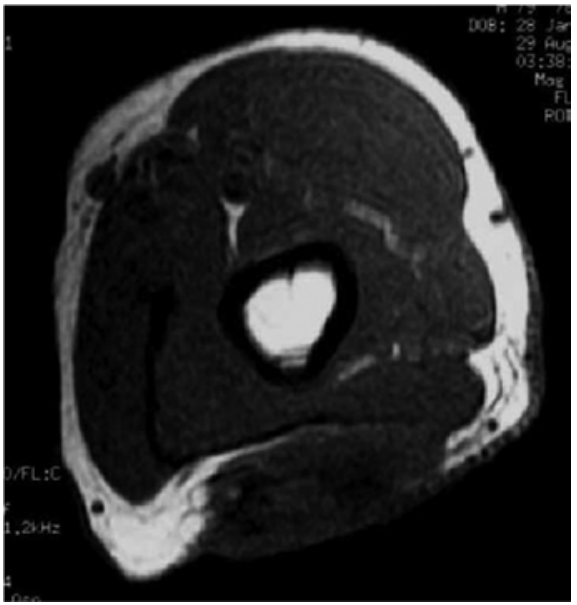

B

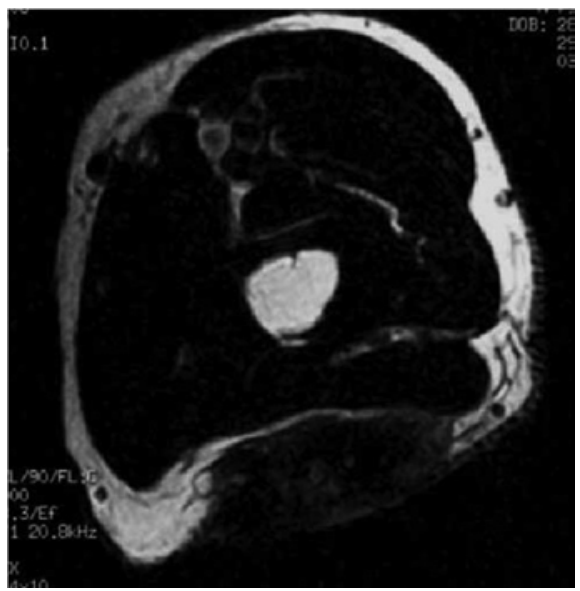

Figure 2. MRI showed a soft-tissue mass above the fascia of the triceps with (A) a low signal intensity on T1-weighted images and (B) a heterogeneous signal intensity on $\mathrm{T} 2$-weighted images.

from 25 to $37 \%$ for ESOS as previously reported $(3,4)$. The tumor size appears to be the only reliable prognostic variable in that tumors greater than $5 \mathrm{~cm}$ have a poor prognosis (3).
A

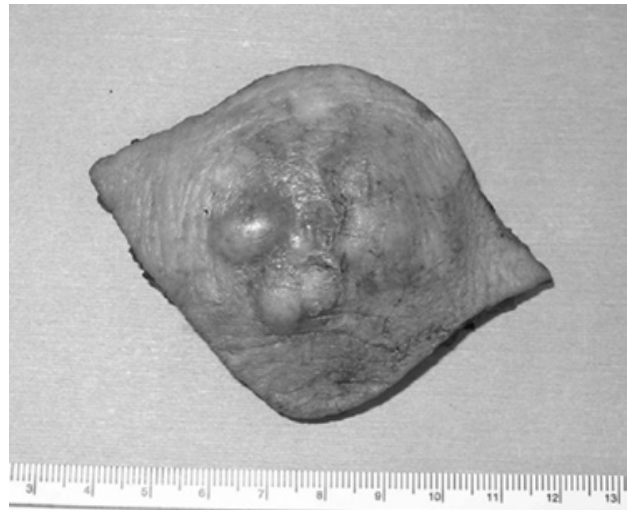

B

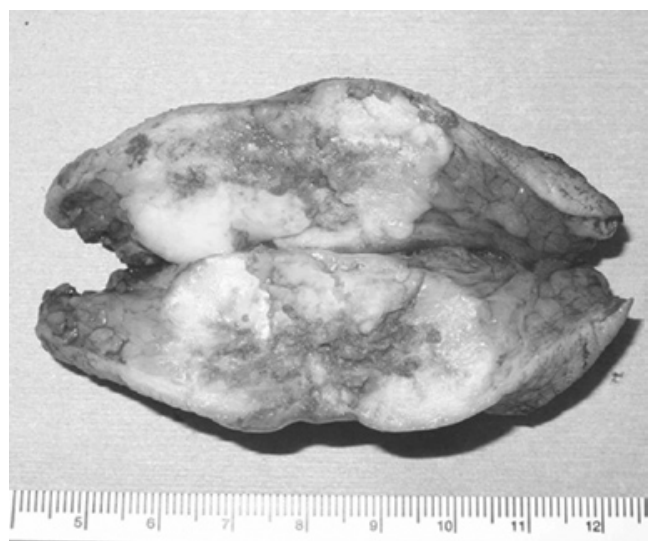

Figure 3. Gross appearance of the tumor (A, surface; B, cut surface). (A) Gross appearance of the resected tumor showed a lobulated hard mass without cutaneous ulceration. (B) The cut surface of the specimen showed a $4 \times 4 \times 2.5 \mathrm{~cm}$ firm and solid mass in the deep dermis and subcutaneous tissue.

A

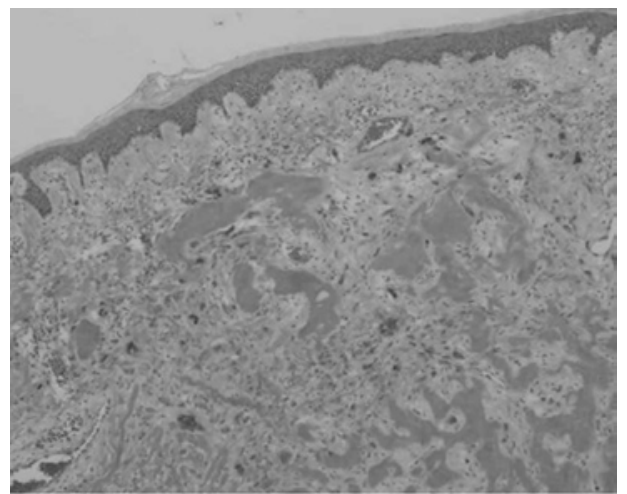

B

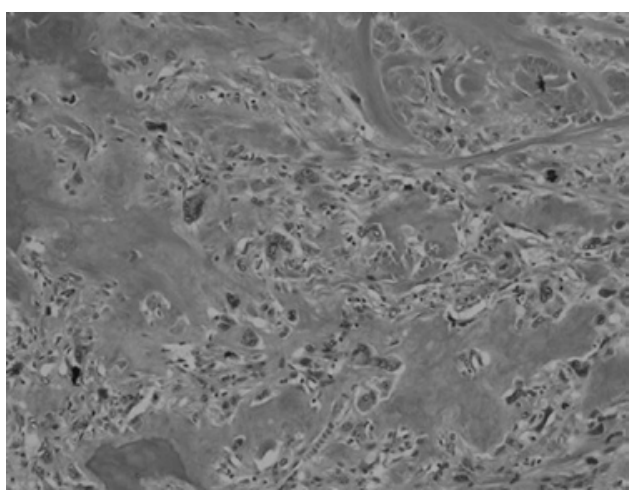

Figure 4. Microscopic findings showed numerous spindle and atypical cells with one or more nuclei, occasional multinucleated giant cells and osteoid and bone formation. H\&E staining (A, magnification, x10; B, magnification, x40). 
Table I. Review of the literature describing cases of primary subcutaneous extraskeletal osteosarcoma.

\begin{tabular}{|c|c|c|c|c|c|}
\hline Author & Age/gender & Location & Size $(\mathrm{cm})$ & Therapy & Outcome \\
\hline Fang et al (8) & 59/female & Abdominal wall & 1.0 & Surgery & CDF, 16 years \\
\hline Yamakage et al (9) & $62 /$ male & Forehead & 3.0 & Surgery & $\begin{array}{l}\text { DOD, } 2 \text { months } \\
\text { (brain metastasis) }\end{array}$ \\
\hline Dubec et al (10) & 75/female & Lower leg & 15.0 & Surgery & CDF, 12 months \\
\hline Pillay et al (11) & $56 /$ male & Scalp & 10.0 & Surgery and chemotherapy & Unknown \\
\hline Oonuma et al (6) & 55/female & Buttock & 1.0 & Surgery and chemotherapy & CDF, 48 months \\
\hline Matsumoto et al (12) & $68 /$ female & Buttock & 1.0 & Surgery & CDF, 16 months \\
\hline Hatano et al (13) & $25 /$ male & Jaw & 1.5 & Surgery and chemotherapy & CDF, 16 months \\
\hline Nakamura et al (Present study) & 79/male & Upper arm & 4.0 & Surgery & CDF, 12 months \\
\hline
\end{tabular}

CDF, continuously disease-free; DOD, dead of disease.

Subcutaneous ESOS was rarely reported. Only eight cases, including the present one, were found in the literature $(6,8-13)$ (Table I). Patients in those studies included 4 males and 4 females, ranging in age from 25 to 79 years. Lesions were located in the buttock in 2 cases and in the scalp, forehead, jaw, abdominal wall, lower leg and upper arm each in 1 case. In general, ESOS develops in the lower extremities, with the thigh being involved most; however, these 8 cases developed ESOS in various anatomical sites. The tumor size was less than $5 \mathrm{~cm}$ in all but 2 cases. A surgical resection was performed in all cases. The consequent surgical margin was wide in 7 patients, including an additional wide resection in 2 cases and an intralesional margin in 1 case. A total of three patients received chemotherapy. A wide surgical resection was performed in the present case. No chemotherapy was administered as a result of the advanced age of the patient. A wide margin is generally recommended for ESOS, as for other high-grade sarcomas (14). Lee et al reported that recurrence is common in ESOS and usually occurs in more than half of the patients (3). However, a wide (or radical) resection should decrease the recurrence of ESOS.

The role of adjuvant chemotherapy in ESOS is unclear. A recent series $(14,15)$ found that the 5-year survival rate of patients with ESOS receiving chemotherapy showed an obvious improvement in comparison to what was described in previous reports $(3,4)$. The two most recent reports found that the 5-year survival rate of patients with chemotherapy was approximately $70 \%(14,15)$. Although adjuvant therapy for ESOS remains controversial, chemotherapy may be useful in an aggressive multimodality approach to this tumor.

The 5-year survival rates associated with ESOS are relatively poor. However, 7 of the 8 cases of subcutaneous ESOS were continuously disease-free. The prognostic significance of the tumor location with respect to its relationship to the superficial fascia of the extremity or trunk was incorporated into the staging system of soft tissue sarcoma in 1998 (16). Although only 9 patients with primary subcutaneous ESOS were previously reported in the literature, these reports may indicate that subcutaneous ESOS has a more favorable prognosis than their more deeply situated counterparts.

\section{References}

1. Allan CJ and Soule EH: Osteogenic sarcoma of the somatic soft tissue. Clinicopathologic study of 26 cases and review of literature. Cancer 27: 1121-1133, 1971.

2. McCarter MD, Lewis JJ, Antonescu CR and Brennan MF: Extraskeletal osteosarcoma: analysis of outcome of a rare neoplasm. Sarcoma 4: 119-123, 2000.

3. Lee JY, Fetsch JF, Wasdhal DA, Lee BP, Pritchard DJ and Nascimento AG: A review of 40 patients with extraskeletal osteosarcoma. Cancer 76: 2253-2259, 1995.

4. Kransdorf MJ and Meis JM: From the archives of the AFIP. Extraskeletal osseous and cartilaginous tumors of the extremities. Radiographics 13: 853-884, 1993.

5. Wilson H: Extraskeletal ossifying tumors. Ann Surg 113: 95-112, 1941.

6. Oonuma M, Hatori M, Hosaka M and Kokubun S: Extraskeletal osteosarcoma arising in the buttock. Upsala J Med Sci 106: 211-215, 2001.

7. Enzinger FM and Weis SW: Osseous soft tissue tumors. In: Soft Tissue Tumors. Enzinger FM and Weiss SW (eds). CV Mosby Company, St. Louis, pp1389-1417, 2001.

8. Fang Z, Yokoyama R, Murai K, Beppu Y and Fukuma H: Extraskeletal osteosarcoma: a clinicopathologic study of four cases. Jpn J Clin Oncol 25: 55-60, 1995.

9. Yamakage A, Kohnoike N, Shiraishi M, et al: A case of extraskeletal osteosarcoma, which has been suspected to be malignant fibrous histiocytoma. Skin Cancer (in Japanese) 11: 161-166, 1996.

10. Dubec JJ, Munk PL, O'Connell JX, et al: Soft tissue osteosarcoma with telangiectatic features: MR imaging findings in two cases. Skeletal Radiol 26: 732-736, 1997.

11. Pillay P, Simango S and Govender D: Extraskeletal osteosarcoma of the scalp. Pathology 32: 154-157, 2000.

12. Matsumoto K, Sakai S, Iijima M, Matsumoto K, Saida T and Kanai S: Subcutaneous tumor on the left buttock. J Jpn Dermatohistopathol Assoc (in Japanese) 19: 18-21, 2003.

13. Hatano $H$, Morita $T$, Kobayashi $H$, Ito $T$, Segawa $H$ and Hasegawa S: Extraskeletal osteosarcoma of the jaw. Skeletal Radiol 34: 171-175, 2005.

14. Torigoe T, Yazawa Y, Takagi T, Terakado A and Kurosawa H: Extraskeletal osteosarcoma in Japan: multiinstitutional study of 20 patients from the Japanese Musculoskeletal Oncology Group. J Orthop Sci 12: 424-429, 2007.

15. Markin HJ, Hornicek FJ, Rosenberg AE, Harmon DC and Gebhardt MC: Survival data for 648 patients with osteosarcoma treated at one institution. Clin Orthop 429: 286-291, 2004.

16. Fleming ID, Phillips JL, Menck HR, Murphy GP and Winchester DP: The National Cancer Data Base report on recent hospital cancer program progress toward complete American Joint Committee on Cancer/TNM staging. Cancer 80: 2305-2310, 1997. 